\title{
Validation of a Wireless Communication Protocol to Monitor Human Gait Using IMUs*
}

\author{
Nuno Ferrete Ribeiro, Joana Figueiredo, and Cristina P. Santos
}

\begin{abstract}
Ambient Assisted Living (AAL) represents a very relevant area of study since it is of great interest to monitor not only people in need of treatment or rehabilitatn, but also healthy and elderly people. One of the main necessities is to monitor the human gait. However, there are still some open issues to be addressed. Namely, literature shows that there are differences between indoor and outdoor environments. Thus, within the framework of a project to monitor human gait using IMUs (Inertial Measurement Units, MPU-6000 Motion Processing Units), the Enhanced Low Power Real Time (eLPRT) protocol was validated by obtaining the percentage of lost packets (lost protocol messages that contains inertial/magnetic sensors information). Concerning the system, the IMUs were connected to a base station (SmartRF05EB) by means of Radio Frequency (RF). This base station was connected to a personal computer to process the data in real time.
\end{abstract}

In order to validate the communication protocol, the losses of data packets were accounted for during the trials carried out by one subject in three different environments: i) inside a laboratory; ii) in a corridor; and iii) in an outdoor environment. As results, the range of average percentage of loss was $0.52 \%$ to $15.21 \%$ inside the laboratory; $1.15 \%$ to $8.93 \%$ in the corridor; and $0.90 \%$ to $7.51 \%$ in the outdoor environment. The absence of ferromagnetic materials and other wireless communications with the same frequency band are the main reasons why the RF transmission had better results in an outdoor environment.

\section{INTRODUCTION}

Sensory systems based on Inertial Measurement Units (IMUs) represent an alternative tool for optical systems, floor sensors or even other wearable sensors systems in the field of gait analysis [1], [2]. These alternative systems reveal an extreme potential to monitor ambulatory activities in the home environment, which is ideal for Ambient Assisted Living (AAL) environments since other methods have spatial constraints [3],[4],[5]. When compared to other systems, IMUs' systems can also be lighter, cheaper or non-invasive [3], [6], [7]. The IMUs' system presented in this article establishes a wireless communication between a base station and IMUs by means of Radio Frequency (RF).

Without considering interferences from other sources or wireless communications, four basic phenomena occur in the wireless medium [8]: i) Path-loss; ii) Reflection; iii) Diffraction; and iv) Scattering. These effects have a negative

\footnotetext{
*This work is supported by the FCT - Fundação para a Ciência e Tecnologia - with the scholarship reference SFRH/BD/108309/2015, with the reference project UID/EEA/04436/2013, by FEDER funds through the COMPETE 2020 - Programa Operacional Competitividade e Internacionalização (POCI) - with the reference project POCI-01-0145FEDER-006941
}

impact on the signal propagation and are responsible for pathloss and the distortion of the received signal. In an indoor environment, the probability of having more reflections is bigger which significantly affects the propagation of the RF signal frequency. In this scenario, the wave partially reflects and partially absorbs if the propagation radio waves reach a surface that is larger than the radio wavelength [8]. On the other hand, the presence of more wireless communications at the same place can also affects the performance of the wireless communication [9]. Concerning the literature [9], to prevent or mitigate the risk of interference, these wireless systems can use: i) Narrow beam adaptive antennas; ii) Power Control; iii) Physical Diversity; and iv) a Monitoring Program.

The primary goal of this article is to check for differences in wireless data transmission between IMUs and the base station in different environments by obtaining the percentage of lost message packets/frames. The remainder of this paper is organized as follows: in Section II the system used by the authors, the communication protocol established between system elements, and validation protocol are presented; Section III presents the results of the communication validation protocol; Section IV provides the discussion for presented results; and Section V contains the conclusion of the current paper.

\section{METHODS AND MATERIALS}

\section{A. Magnetic/Inertial-based Measurement System}

As depicted in Fig. 1, Magnetic/Inertial-based Measurement System (IMUs' System) contains three elements: a personal computer (PC); a SmartRF05EB base station; and the sensory modules. The base station is equipped with a CC2530EM (Evaluation Module) from Texas Instruments (IEEE Std 802.15.4, 2006), and has the responsibility to associate new sensory modules to the network, allocate time slots on the Enhanced Low Power Real Time (eLPRT) superframe for modules to transmit, and keep the synchronization in the network [10].

Each sensory module is constituted by a sensor board that contains a MPU-6000 [11] from InvenSense ${ }^{\circledR}$ (InvenSense, Inc., US), and a 3.6 Volts battery. In turn, each MPU-6000 is formed by a three-axis MEMS accelerometer and gyroscope, a Honeywell three-axis Digital Compass IC HMC5883L [12], and a temperature sensor. The sensor board is connected to a

N. F. Ribeiro, J. Figueiredo, and C. Santos are with the Center of MicroElectroMechanical Systems (CMEMS), University of Minho, Guimarães, Portugal. E-mails: \{a68359@alunos; id6003@alunos; cristina@dei\}.uminho.pt. 
CC2530EM module through two 20 pin headers [13]. To briefly summarise, wireless communication between the sensory module and the base station is made using CC2530 modules. The base station sends the received data to the PC by serial port. Subsequently, the data is processed in real time. The sampling frequency was $30 \mathrm{~Hz}$.

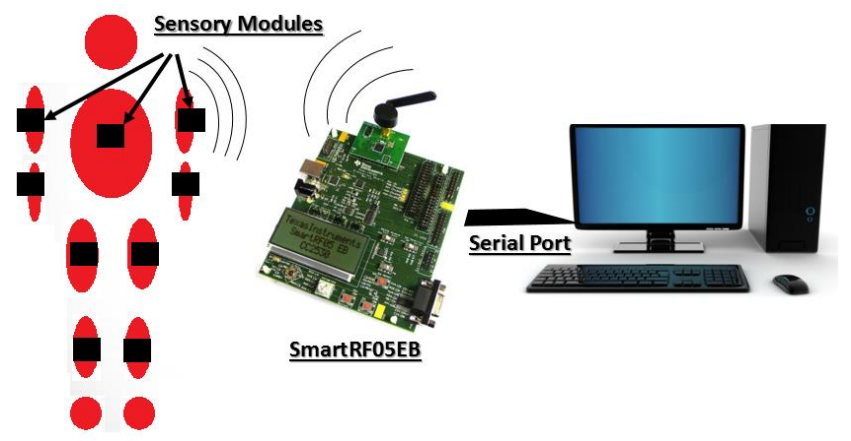

Figure 1. Magnetic/Inertial-based Measurement System elements.

\section{B. eLPRT Protocol}

The Enhanced Low Power Real Time (eLPRT) protocol controls the communication through the wireless medium, and was designed to optimize the quality of service (QoS) support and the bandwidth utilization efficiency [10]. In order to transmit the multiple sensor readings between the sensory modules and the base station, a multi-byte message is formed after the sensors readings have been collected and converted to digital through a 16-bit ADC. This message, depicted in Fig. 2.a, will be designated from now on as frame. Each reading is expressed in two bytes for each axis of each used sensor. In Fig. 2.b, "S1 Acc_X1" represents the first byte from the first accelerometer reading/sample, and "S1 Acc_X2" the second byte from the same reading. This strategy is also applied for the remaining axes and other samples. The "Type" byte (Fig. 2.a) indicates if the message is a command message or a sensors data message. The "Length" byte gives information about the size of the payload. The CRC (cyclic redundancy check) values ( $\mathrm{CRC} 1$ and $\mathrm{CRC} 2$ ) are used to detect accidental changes to raw data. If they do not match, then the block contains a data error.

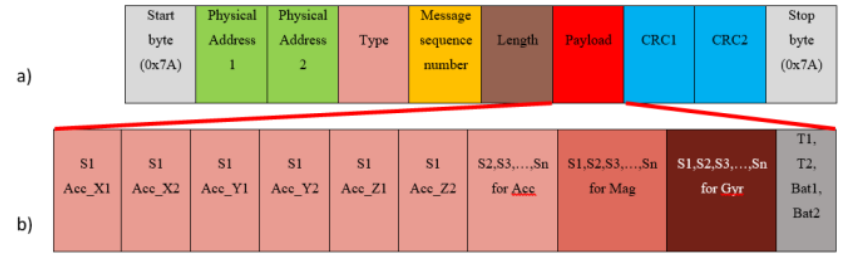

Figure 2. a) Constitution of the frame; b) Constitution of the payload (Ssample/reading, T1 \& T2-temperature byte 1 and 2, Bat1 \& Bat2-battery byte 1 and 2).

Once frames reach the base station, they are processed in order to obtain relevant information from sensory modules correctly. This process must be fast and efficient. For instance, two equal frames, i.e., two equal sequence numbers, should not be processed. Thus, an Algorithmic State Machine (ASM) was implemented to improve the reliability of the data processing where the frame's constitution is taken into account. This ASM showed an efficiency of $100 \%$ for a use of 500 times.

\section{Validation Protocol}

A healthy male subject with 22 years old $(1.83 \mathrm{~m} ; 63 \mathrm{~kg})$ performed two different trials in three different environments: i) inside a laboratory; ii) in a corridor; and iii) outdoor environment free of any ferromagnetic influence or other wireless communications. In the first trial, the participant stood upright for 20 seconds at one meter from base station, while in the second trial, he stood upright for 5 seconds and then walk forward 5 meters (inside a laboratory) and 10 meters (in the other two environments) at two different paces (normal and fast). In both scenarios, after the test was completed, the subject turned off the sensory modules. The losses in this period of time were also accounted for until all sensory modules were disabled.

The first mentioned trial (Trial I) was performed only with five sensory modules as depicted in Fig. 3.a. In the second trial (Trial II), the subject was asked to carry out several experiments wearing 1, 2, 3, 4, and 5 sensory modules. For each environment, these two trials were repeated five times per each spatial arrangement of the sensory modules in the body as depicted in Fig. 3.

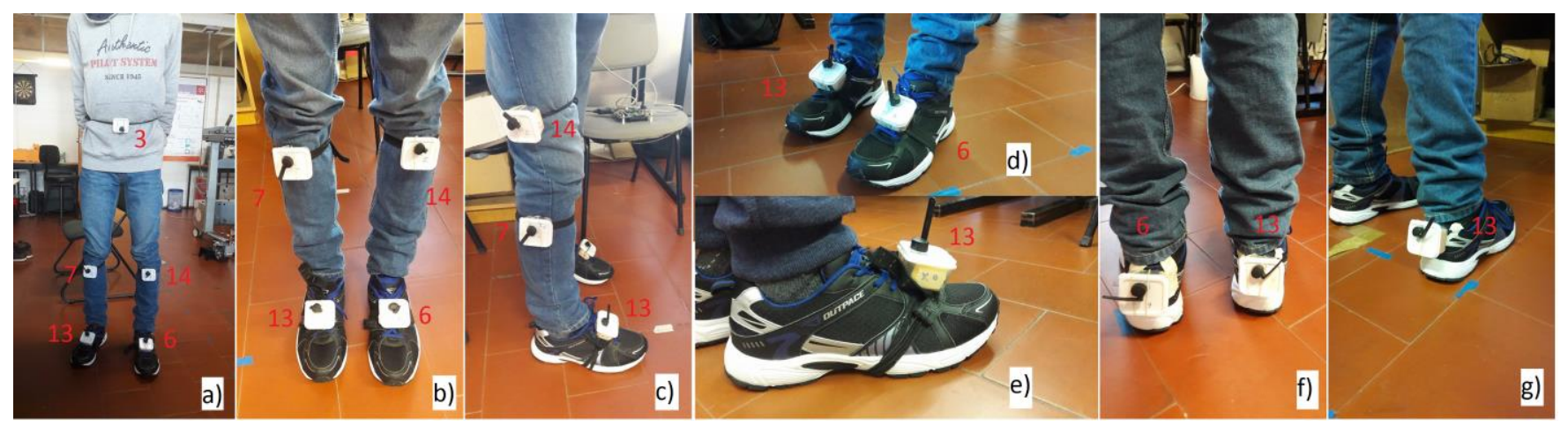

Figure 3. Spatial arrangement of the sensory modules in the body with their physical addresses: a) 5 sensory modules on trunk (3), foot (13-right, 6-left), and shank (7-right, 14-left); b) 4 sensory modules on foot (13-right, 6-left), and shank (7-right, 14-left); c) 3 sensory modules on right leg: foot (13), shank (7), and thigh (14); d) 2 sensory modules on the upper foot (13-right, 6-left); e) 1 sensory module on the upper foot (13-right); f) 2 sensory modules on the heel (13-right, 6-left); and g) 1 sensory module on the heel (13-right). 


\section{RESULTS}

\section{A. Trial I}

The average percentage of loss of the five tests was $3.40 \pm 2.59 \%$ inside the laboratory, $2.03 \pm 0.97 \%$ in the corridor, and $0.28 \pm 0.07 \%$ in the outdoor environment. Table I details the number of losses verified in each test, as well as the total number of frames received during the trial. In this trial, the number of lost frames is always less than 5 in an outdoor environment. On the other hand, the number of lost frames is greater than 10 in every experiment carried out inside the laboratory and in the corridor.

TABLE I. NUMBER OF LOST AND TOTAL FRAMES FOR EACH ENVIRONMENT

\begin{tabular}{|c|c|c|c|c|c|c|}
\hline \multirow{2}{*}{ Test } & \multicolumn{3}{|c|}{ Number of Lost frames } & \multicolumn{3}{c|}{ Number of Total frames } \\
\cline { 2 - 7 } & Lab & Corridor & Outdoor & Lab & Corridor & Outdoor \\
\hline 1 & 57 & 10 & 3 & 1167 & 1157 & 1157 \\
\hline 2 & 19 & 37 & 4 & 1234 & 1174 & 1176 \\
\hline 3 & 26 & 32 & 4 & 1194 & 1175 & 1147 \\
\hline 4 & 85 & 14 & 3 & 1174 & 1158 & 1145 \\
\hline 5 & 14 & 26 & 2 & 1200 & 1190 & 1130 \\
\hline
\end{tabular}

\section{B. Trial II}

The second trial was performed seven times since this was done for all forms of spatial arrangement of the sensory modules described above in Fig. 5. Table II summarizes the values of the average percentage of loss of the five tests for each spatial arrangement in the three mentioned environments at different paces. Table II discloses the average percentage of loss per each spatial arrangement in different environments at normal and fast pace. This values are also represented in Figs. 4 to 6 (one figure per environment: Lab, Corridor, and Outdoor, respectively) to make an easy to understand comparison between normal and fast pace for each spatial arrangement of the sensory modules. Moreover, as results, for self-comfortable gait speed the subject performed a mean velocity of $3.24 \pm 0.17 \mathrm{~km} / \mathrm{h}$, and for fast pace the tests mean velocity was $6.04 \pm 0.34 \mathrm{~km} / \mathrm{h}$.

TABLE II. PERCENTAGE OF LOSS OF THE FIVE TESTS FOR EACH SPATIAL ARRANGEMENT (L-LAB; C-CORRIDOR; O-OUTDOOR; N-NORMAL PACE; F- FAST PACE; UF-ON THE UPPER FOOT; H-ON THE HEEL)

\begin{tabular}{|c|c|c|c|c|c|c|}
\hline \multirow{2}{*}{$\begin{array}{c}\text { Spatial } \\
\text { Arr. }\end{array}$} & \multicolumn{3}{|c|}{ \% of Loss - N } & \multicolumn{3}{c|}{ \% of Loss - F } \\
\cline { 2 - 7 } & Lab & Corridor & Outdoor & Lab & Corridor & Outdoor \\
\hline \multirow{2}{*}{1 -UF } & 0.78 & 3.57 & 1.07 & 1.68 & 3.06 & 1.19 \\
& \pm 0.25 & \pm 2.09 & \pm 0.41 & \pm 0.87 & \pm 1.58 & \pm 0.60 \\
\hline \multirow{2}{*}{2 -UF } & 0.59 & 1.15 & 1.36 & 0.52 & 1.32 & 1.87 \\
& \pm 0.19 & \pm 0.86 & \pm 1.49 & \pm 0.15 & \pm 0.81 & \pm 0.61 \\
\hline \multirow{2}{*}{1 -H } & 1.31 & 1.37 & 0.90 & 1.64 & 2.52 & 0.90 \\
& \pm 0.11 & \pm 0.72 & \pm 0.35 & \pm 1.27 & \pm 1.58 & \pm 0.35 \\
\hline \multirow{2}{*}{2 -H } & 0.74 & 3.46 & 1.18 & 0.87 & 1.72 & 1.36 \\
& \pm 0.71 & \pm 2.17 & \pm 0.64 & \pm 0.49 & \pm 0.37 & \pm 0.42 \\
\hline \multirow{2}{*}{3} & 9.86 & 4.28 & 0.90 & 8.24 & 5.43 & 1.32 \\
& \pm 3.46 & \pm 2.33 & \pm 0.60 & \pm 5.35 & \pm 2.89 & \pm 0.58 \\
\hline \multirow{2}{*}{4} & 5.82 & 6.72 & 3.14 & 12.64 & 3.84 & 4.85 \\
& \pm 3.87 & \pm 4.94 & \pm 1.84 & \pm 5.87 & \pm 1.87 & \pm 2.81 \\
\hline \multirow{2}{*}{5} & 15.21 & 8.93 & 5.40 & 9.74 & 5.80 & 7.51 \\
& \pm 4.04 & \pm 5.41 & \pm 2.04 & \pm 2.82 & \pm 3.27 & \pm 2.07 \\
\hline \multirow{2}{*}{ Mean } & 4.90 & 4.21 & 1.99 & 5.05 & 3.38 & 2.71 \\
& \pm 1.81 & \pm 2.65 & \pm 1.05 & \pm 2.40 & \pm 1.77 & \pm 1.06 \\
\hline
\end{tabular}

In Fig. 4, the average percentage of loss inside the laboratory for 2 or less sensory modules is relatively low. However, when the number of sensory modules is equal to or greater than three, the average percentage of loss is always bigger than $5 \%$. In some cases, this percentage is bigger than $10 \%$ which can affect the gait monitoring process.

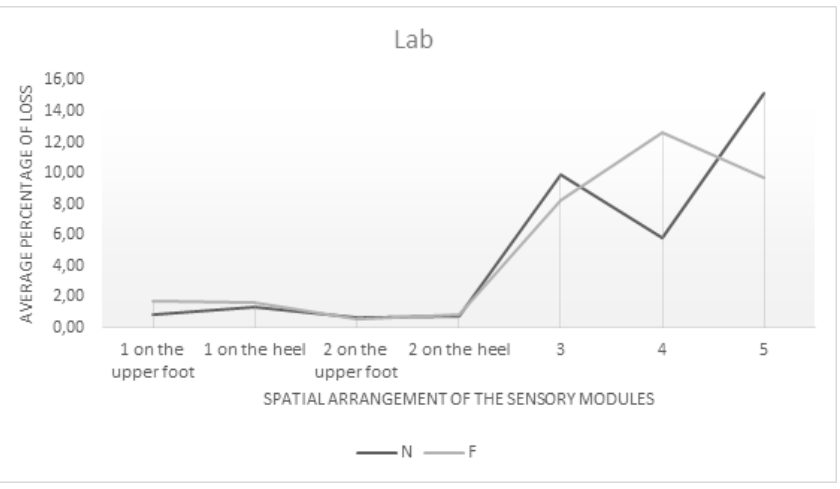

Figure 4. Average percentage of loss for each spatial arrangement in laboratory (N-Normal pace; F- Fast pace).

In general, at the corridor, the average percentage of loss (Fig. 5) is lower than inside the laboratory. None of the means is greater than $8 \%$. In this case, when the number of sensory modules is 3 , the average percentage of loss is lower than $6 \%$, which is inferior to the value in the previous situation $(8.24 \%$ at fast pace, and $9.86 \%$ at normal pace). Similar finding is observed when 4 or 5 sensory modules were used. However, in the situation where one sensory module was used, the result was higher in comparison to the result evidenced in Fig. 4. This also happens when 2 sensory modules were attached on the heel and on the upper foot.

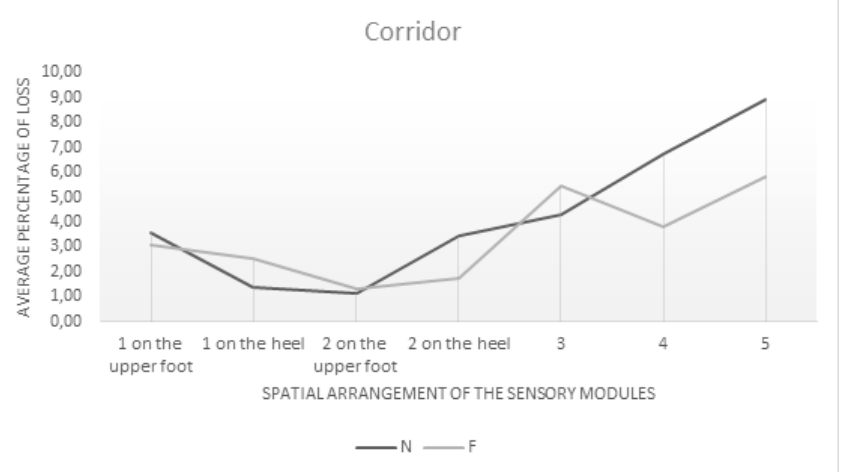

Figure 5. Average percentage of loss for each spatial arrangement in the corridor (N-Normal pace; F- Fast pace).

In an outdoor environment, the average percentage of loss (Fig. 6) is lower than $2 \%$ when 3 or fewer sensory modules were used. When the subject wore 4 and 5 sensory modules, the average percentage of loss was lower than, $5 \%$ and $8 \%$, respectively. In general, the developed protocol for the wireless communication of various sensory modules based on IMUs, was more efficient and robust in outdoor than inside the laboratory or in the corridor. In Table II, in the "Mean" line, the average percentage values are accompanied by the mean of the standard deviations of the various sensory modules spatial arrangements (Fig. 3). 


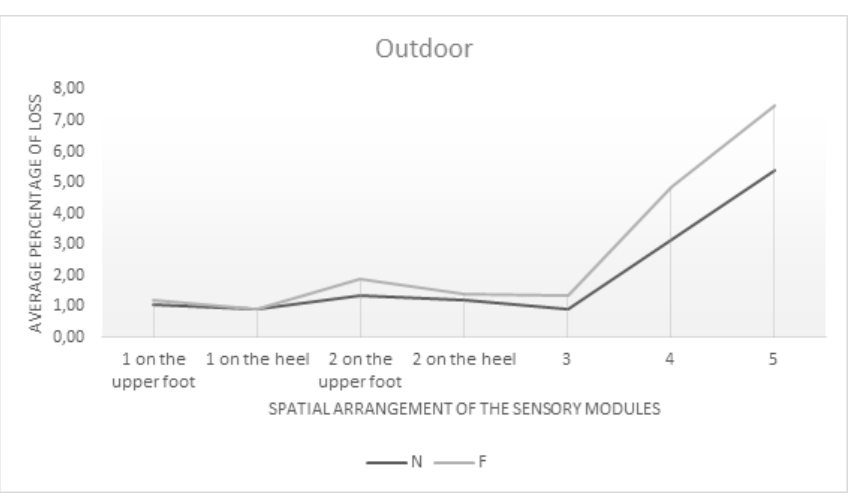

Figure 6. Average percentage of loss for each spatial arrangement in an outdoor environment (N-Normal pace; F- Fast pace).

\section{DISCUSSION}

Regarding the first trial, a better result was expected in the outdoor environment, since it is an environment free of any wireless communications like those existing in the laboratory and in the corridor. In fact, the average percentage of loss in the outdoor environment was lower than the one observed in the other environments (Outdoor- 0.28 $\pm 0.07 \%$; Lab$3.40 \pm 2.59 \%$; Corridor $-2.03 \pm 0.97 \%$ ), as expected. In addition, in the corridor the losses are slightly lower than those found inside the laboratory, which means that any interferences in these two environments have greater influence inside the laboratory than in the corridor.

The results of the second trial results reveal that as the number of modules increases, the percentage of loss also increases. However, once again and for the same reasons the average percentage of loss is smaller in an outdoor environment than in the corridor and inside the laboratory, and results in the corridor are better than inside the laboratory. Taking into account the graphs depicted on Fig. 4, Fig. 5, and Fig. 6, it is possible to retain that the average percentage of loss when the subject performed normal and fast speed shows the same trend in both scenarios for every environment. In other words, the gait speed has almost no influence on the loss of frames. However, in the majority of the spatial arrangements of the sensory modules, it was found that there is a slightly higher average percentage of loss when the subject walked at a fast speed. Although not significant, this may be justified by the fact that during a more sudden movement, the connection between the sensor board and the CC2530 module may fail.

The first trial can be compared to the second trial when the subject used 5 sensory modules (Fig. 3.a). Thus, it is possible to verify that when the subject walked, independently of the gait speed, the average percentage of loss was higher than the homologous value recorded when the subject was standing upright at one meter from the base station. This increase is explained by the gradual increase in the distance between the sensory modules and the base station. When the distance increases, the risk of interferences, reflections, diffractions, and scattering also increases.

\section{CONCLUSION}

According to this experiment, it is consensual that the presence of others wireless communications affect the good performance of the system. So, in order to overcome this difficulty, it would be advantageous to change the frequency band of the communication protocol depending on the location where the data is collected. Therefore, an unused frequency band or a frequency band in which its signal is weak should be chosen.

The distance in indoor environments between sensory modules and the base station can also affects the number of lost frames. As a future work it is suggested to execute trials with the base station and the sensory modules in different indoor rooms.

The presence of electrically conductive metals inside the laboratory can reflect and absorb the radio waves and consequently it interfere their transmission. This problematic can also be a reason why so many frames were lost in this environment and in the corridor. Perhaps a future study that focus on the interference of conductive metals in this system may allow meeting new limitations, as well as new resolutions of existing problems.

\section{ACKNOWLEDGMENT}

N. F. R., J. F., and C. P. S. thank Hélder Silva, Pedro Macedo, and Luís Rocha from Center of MicroElectroMechanical Systems (CMEMS) for their support in this paper.

\section{REFERENCES}

[1] A. Muro-de-la-Herran et al., "Gait analysis methods: An overview of wearable and non-wearable systems, highlighting clinical applications," Sensors, v. 14, no. 2, pp. 3362-3394, 2014.

[2] J. Favre, R. Aissaoui, B. M. Jolles, J. A. de Guise, and K. Aminian, "Functional calibration procedure for 3D knee joint angle description using inertial sensors," J. Biomech., v. 42, no. 14, pp. 2330-2335, 2009.

[3] T. Liu et al., "Development of a wearable sensor system for quantitative gait analysis," Measurement, v. 42, no. 7, pp. 978-988, 2009.

[4] S. T. Boerema, L. Van Velsen, L. Schaake, T. M. Tonis, and H. J. Hermens, "Optimal Sensor Placement for Measuring Physical Activity with a 3D Accelerometer," Sensors, v. 14, pp. 3188-3206, 2014.

[5] E. Campo, S. Bonhomme, M. Chan, and D. Esteve, "Remote tracking patients in retirement home using wireless multisensor system," in eHealth Networking Applications and Services (Healthcom), 2010 12th IEEE International Conference on, 2010.

[6] L. Ambrozic, M. Gorsic, S. Slajpah, R. Kamnik, and M. Munih, "Wearable Sensory System for Robotic Prosthesis," pp. 269-275, 2013

[7] S. Tadano, R. Takeda, and H. Miyagawa, “Three Dimensional Gait Analysis Using Wearable Acceleration and Gyro Sensors Based on Quaternion Calculations," Sensors, v. 13, pp. 9321-9343, 2013.

[8] M. Kochlán et al., "2.4GHz ISM Band Radio Frequency Signal Indoor Propagation," in Federated Conference on Computer Science and Information Systems, 2014, v. 2, pp. 1027-1034.

[9] M. Fitzmaurice, "Use of $2.4 \mathrm{GHz}$ Frequency Band for Communications Based Train Control Data Communication Systems," in Joint Rail Conference, 2006, pp. 263-267.

[10] J. A. Afonso, H. D. Silva, P. Macedo, and L. A. Rocha, "An enhanced reservation-based MAC protocol for IEEE 802.15.4 networks," Sensors, v. 11, no. 4, pp. 3852-3873, 2011.

[11] InvenSense, "MPU-6000 and MPU-6050 Product Specification," 2013.

[12] Honeywell International Inc., "3-Axis Digital Compass IC HMC5883L," 2011.

[13] P. Macedo, J. A. Afonso, L. A. Rocha, and R. Simoes, "A Telerehabilitation System Based on Wireless Motion Capture Sensors," in PhyCS - Proceedings of the International Conference on Physiological Computing Systems, 2014, pp. 55-62. 